\title{
The effect of ethanol concentration on the morphological and molecular preservation of insects for biodiversity studies
}

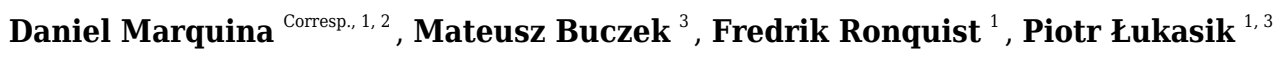 \\ 1 Department of Bioinformatics and Genetics, Swedish Museum of Natural History, Stockholm, Sweden \\ 2 Department of Zoology, Stockholm University, Stockholm, Sweden \\ 3 Institute of Environmental Sciences, Faculty of Biology, Jagiellonian University Cracow, Krakow, Poland \\ Corresponding Author: Daniel Marquina \\ Email address: daniel.marquina@nrm.se
}

Traditionally, insects collected for scientific purposes have been dried and pinned, or preserved in $70 \%$ ethanol. Both methods preserve taxonomically informative exoskeletal structures well but are suboptimal for preserving DNA for molecular biology. Highly concentrated ethanol (95 - $100 \%$ ), preferred as a DNA preservative, has generally been assumed to make specimens brittle and prone to breaking. However, systematic studies on the correlation between ethanol concentration and specimen preservation are lacking. Here, we tested how preservative ethanol concentration in combination with different sample handling regimes affect the integrity of seven insect species representing four orders, and differing substantially in the level of sclerotization. After preservation and treatments (various levels of disturbance), we counted the number of appendages (legs, wings, antennae, or heads) that each specimen had lost. Additionally, we assessed the preservation of DNA after long-term storage by comparing the ratio of PCR amplicon copy numbers to an added artificial standard. We found that high ethanol concentrations indeed induce brittleness in insects. However, the magnitude and nature of the effect varied strikingly among species. In general, ethanol concentrations at or above $90 \%$ made the insects more brittle, but for species with robust, thicker exoskeletons, this did not translate to an increased loss of appendages. Neither freezing the samples nor drying the insects after immersion in ethanol had a negative effect on the retention of appendages. However, the morphology of the insects was severely damaged if they were allowed to dry. We also found that DNA preserves less well at lower ethanol concentrations when stored at room temperature for an extended period. However, the magnitude of the effect varies among species; the concentrations at which the number of COI amplicon copies relative to the standard was significantly decreased compared to $95 \%$ ethanol ranged from $90 \%$ to as low as $50 \%$. While higher ethanol concentrations positively affect long-term DNA preservation, there is a clear trade-off between preserving insects for morphological 
examination and genetic analysis. The optimal ethanol concentration for the latter is detrimental for the former, and vice versa. These trade-offs need to be considered in large insect biodiversity surveys and other projects aiming to combine molecular work with traditional morphology-based characterization of collected specimens. 
1 The effect of ethanol concentration on the morphological and molecular preservation of insects for 2 biodiversity studies

3

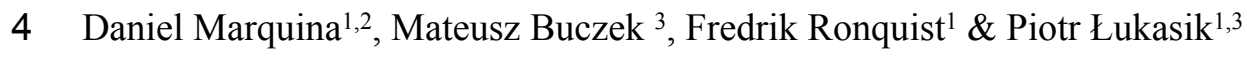

$5{ }^{1}$ Department of Bioinformatics and Genetics, Swedish Museum of Natural History, Stockholm, Sweden.

$6 \quad{ }^{2}$ Department of Zoology, Stockholm University, Stockholm, Sweden.

$7 \quad{ }^{3}$ Institute of Environmental Sciences, Jagiellonian University, Krakow, Poland

8

9 Corresponding author: Daniel Marquina. Address: Department of Bioinformatics and Genetics, Swedish 10 Museum of Natural History, Stockholm, Sweden; Department of Zoology, Stockholm University, 11 Stockholm, Sweden. Email: daniel.marquina@nrm.se

12

13 Running title: Insect fragility and DNA preservation in ethanol 14 
16 Abstract

17 1. Traditionally, insects collected for scientific purposes have been dried and pinned, or preserved in $70 \%$

18 ethanol. Both methods preserve taxonomically informative exoskeletal structures well but are suboptimal

19 for preserving DNA. Highly concentrated ethanol (95 - $100 \%)$, preferred as a DNA preservative, has

20 generally been assumed to make specimens brittle and prone to breaking. However, systematic studies on

21 the correlation between ethanol concentration and specimen preservation are lacking.

22 2. We tested how preservative ethanol concentration in combination with different sample handling 23 regimes affect the integrity of seven insect species representing four orders, and differing substantially in 24 the level of sclerotization. After preservation and treatments (various levels of disturbance), we counted 25 the number of appendages (legs, wings, antennae, heads) that specimens had lost. Additionally, we assessed the preservation of DNA after long-term storage by comparing the ratio of PCR amplicon copy numbers to an added artificial standard.

3. We found that high ethanol concentrations indeed induce brittleness in insects. However, the magnitude and nature of the effect varied strikingly among species. In general, ethanol concentrations at or above $90 \%$ made the insects more brittle, but for species with robust, thicker exoskeletons, this did not translate to an increased loss of appendages. Neither freezing nor drying the insects after immersion in ethanol had a negative effect on the retention of appendages. We also found that DNA preserves less well at lower ethanol concentrations when stored at room temperature for an extended period. However, the magnitude of the effect varies among species; the concentrations at which the number of COI amplicon copies relative to the standard was significantly decreased compared to $95 \%$ ethanol ranged from $90 \%$ to as low as $50 \%$.

4. While higher ethanol concentrations positively affect long-term DNA preservation, there is a clear trade-off between preserving insects for morphological examination and genetic analysis. The optimal ethanol concentration for the latter is detrimental for the former, and vice versa. These trade-offs need to be considered in large insect biodiversity surveys and other projects aiming to combine molecular work 41 with traditional morphology-based characterization of collected specimens. 
43 Keywords: insects, ethanol, DNA preservation, morphology, collections

44

45

\section{Introduction}

47 The first records of the use of ethanol for the preservation of animal tissue date back to the mid $1600 \mathrm{~s}$, 48 when Robert Boyle mentions that he successfully used the "Spirit of Wine" to preserve blood and soft parts of a human body, as well as a fish, for many months (Boyle, 1664). The knowledge of the preservative properties of ethanol, in combination with the discovery of cheap and effective ways of producing ethanol in high concentration, led to widespread adoption among naturalists. Consequently, ethanol has been the fixative most widely used in museum and private natural history collections since the $18^{\text {th }}$ century. The attractiveness of ethanol for other human uses has sometimes caused challenges, however. For instance, Carl Linnæus, the father of modern taxonomy, needed to apply for a special permission to import ethanol for preserving his collections after home distilling was temporarily banned in Sweden (von Linné, 1764).

7 During the last two decades, as the research focus has shifted from the analysis of morphological features to molecular work, including DNA sequencing and amplification, ethanol has remained the preferred preservative liquid. Ethanol is an excellent fixative for DNA for three reasons: it kills decomposing microorganisms; it removes water from the tissues, slowing down enzymatic processes; and it denatures both the DNA and DNA-degrading enzymes, preventing further enzymatic degradation (Srinivasan, Sedmak, \& Jewell, 2002).

The standard ethanol concentration employed for preserving insects for morphological examination used to be $70 \%$ (Martin, 1977). However, a higher concentration (95\% or higher) has been recommended for the optimal preservation of DNA (Nagy, 2010). Although $70 \%$ or $80 \%$ ethanol is known to suffice as a DNA preservative for PCR and sequencing purposes during short-term storage (Carew, Coleman \& Hoffman, 2018; Stein, White, Mazor, Miller \& Pilgrim, 2013), it has been shown 
68 that DNA degradation occurs in the longer term, resulting in the molecule getting increasingly fragmented

69 over time (Baird, Pascoe, Zhou \& Hajibabaei, 2011; Carew, Metzeling, Clair \& Hoffman, 2017). Thus, it

70 would appear advantageous to change the standard practice and preserve insects in higher ethanol

71 concentrations. This idea has not been universally adopted, however, because of the common assumption

72 that high grade ethanol makes the insects brittle and prone to damage during manipulation, most likely

73 due to excessive tissue dehydration. However, to our knowledge, there is only one published study that

74 has directly addressed the effect of high ethanol concentrations on insect preservation for morphological

75 study: King \& Porter (2004) used three species of ants to test the effects of ethanol concentration on the

76 mounting of specimens, concluding that $95 \%$ ethanol made the ants hard to mount and prone to breaking.

77 Our own experience with other insect groups is similar. For instance, we noted that cicadas (Hemiptera)

78 become very brittle when preserved in $95 \%$ ethanol (repeatedly replaced to prevent its dilution by insect

79 body fluids) and subsequently mounted. Preserving them in $90 \%$ ethanol made cicadas much more

80 suitable for mounting and taxonomic characterization (K. Nazario and P. Łukasik, pers. obs).

81 Increasingly, insect biodiversity inventories and ecological assessments are accompanied by or solely

82 rely on DNA sequencing (e.g., Janzen et al., 2009; Shokralla et al., 2014; see Matos-Maraví et al. (2019)

83 for a comprehensive review on insect genomics applied to biodiversity study). This leads researchers to

84 collect samples in the field in higher concentration ethanol, which could be detrimental for the

85 morphological integrity of the insects collected, potentially causing the loss of morphological characters

86 and decreasing the value of the specimens for taxonomic description or further anatomical or ecological

87 study. When the purpose is to store specimens for both DNA sequencing and morphological study,

88 researchers seem to be using a variety of approaches: from standardizing ethanol concentrations to $80 \%$,

89 through opting for concentrations closer to $95 \%$, to starting with a high concentration and not measuring

90 it again after insects are preserved, or relying on sub-zero temperatures as the primary means of

91 preservation. There appears to be no hard evidence or consensus on which approach is optimal.

92 While it may be challenging to find a sweet spot that is acceptable for all intended uses, there is a clear 93 need for experimental studies of the nature of the trade-offs between the preservation of insects for DNA 
94 sequencing and morphological study. Here, we take a few steps towards filling this knowledge gap.

95 Specifically, we examined the effects of increasing ethanol concentrations on specimen fragility and DNA

96 preservation in seven species of insects, spanning four orders and representing different sizes and levels

97 of sclerotization.

98

99

100 Materials and Methods

101 Mock communities

102 For the experiments, we constructed artificial (mock) communities made up of seven species of insects

103 (Figure 1A): Macrolophus pygmaeus (Hemiptera: Miridae), Aphidoletes aphidimyza (Diptera:

104 Cecidomyiidae), Drosophila hydei (Diptera: Drosophilidae), Dacnusa sibirica (Hymenoptera:

105 Braconidae), Calliphora vomitoria (Diptera: Calliphoridae), Formica rufa (Hymenoptera: Formicidae)

106 and Dermestes haemorrhoidalis (Coleoptera: Dermestidae). For better readability, only the generic names

107 will be used from now on in the article. Adults of these species represent a wide range of body shapes,

108 cuticle hardness levels, and responses to varying ethanol concentrations and treatments based on

109 anecdotal information and prior observations. Specimens of some species were commercially purchased,

110 and others were manually collected (Table S1). Specimens of Macrolophus, Drosophila, Dacnusa,

111 Calliphora, and Formica were first killed by freezing them at $-20{ }^{\circ} \mathrm{C}$ for $1-2 \mathrm{~h}$ and then placed in the

112 experimental tubes at the desired ethanol concentration (see below). Specimens of Aphidoletes and

113 Dermestes were killed by placing them in the experimental tubes at the desired concentrations directly.

114 All mock communities consisted of ten individuals of Macrolophus, Aphidoletes, Drosophila and

115 Dacnusa, and two individuals of Calliphora, Formica, and Dermestes. The specimens were kept in 50

$116 \mathrm{~mL}$ Falcon tubes with $40 \mathrm{~mL}$ of preservative ethanol of different concentrations. Communities were

117 prepared over the course of approx. 2 weeks. Once all communities were ready, the ethanol was replaced

118 with a fresh aliquot of ethanol at the same concentration and kept for a month at room temperature to

119 standardize the incubation before the treatments began.

Peer) reviewing PDF | (2020:11:55112:1:0:NEW 23 Dec 2020) 
We used these preserved mock communities for three experiments, where we measured the effect of

121 ethanol concentration on the morphological integrity of specimens subjected to different handling

122 regimes, as described below. Insects used for the first of these experiments were subsequently stored

123 long-term at the same ethanol concentration and used for the DNA preservation study.

124

Experiment 1: Effect of ethanol concentration on specimen brittleness

126 In the first experiment, we analyzed whether short-term preservation in high concentrations of ethanol 127 alone increased the fragility of the insects. For the main experiment (Figure 1B), we used mock 128 communities preserved in eight ethanol concentrations: 30, 50, 70, 80, 90, 95, 97, and 99\%, with ten replicate tubes for each concentration. The tubes were subjected to two different shaking regimes by manually vortexing them in a horizontal position for either $1 \mathrm{~min}$ ("Gentle" shaking, five tubes per concentration) or 2 min ("Vigorous" shaking, the remaining five tubes per concentration). After each treatment, all individuals were inspected under a stereomicroscope and scored for lost appendages. The type of appendages scored for each species varied, and this was decided based on pilot trials (see

134 Supplementary Material). Specifically, we scored the loss of: head, legs, wings, and antennae for Macrolophus, Aphidoletes, and Dacnusa; head, legs, and wings for Drosophila; legs and wings for Calliphora and Dermestes; and legs and antennae for Formica. Only forewings were considered (elytra in 137 the case of Dermestes).

140 In the second experiment, we measured if the sensitivity to transport-induced damage was influenced by

141 the ethanol concentration. For this experiment (Figure 1C), we used two ethanol concentrations: 70 and $14295 \%$, with 12 mock community replicates in each. These tubes were transported in three ways, 143 corresponding to treatments. Treatment "Walking" consisted of the experimenter (D.M.) carrying three

144 tubes/concentration in the backpack home-to-work and work-to-home for a total distance of approx. 10 $145 \mathrm{~km}$ (three days * $3.2 \mathrm{~km}$ ), walking only. Treatment "Running" was similar, but the experimenter (P.t.) 
146 ran, chasing after public transportation, for approximately half of the total distance of approx. $8 \mathrm{~km}$ (three

147 days * $2.7 \mathrm{~km}$ ). The last treatment, "PostNord", consisted of two shipments of 3 tubes/concentration,

148 separated by one week, from the Swedish Museum of Natural History in Stockholm to Station Linné in

149 Öland and back, using the Swedish national post service. We reasoned that these three treatments would

150 represent the range of handling regimes that insect samples collected in the field might experience in

151 practice. Damage induced to the individual insects was assessed as above.

152

153 Experiment 3: Effect of storage and processing factors

154 In the third experiment, we tested if other types of treatment can magnify the effects of high ethanol

155 concentrations on insect brittleness (Figure 1D). For each of the two ethanol concentrations tested (70 and

$15695 \%$ ), we used 12 replicate mock communities, four tubes/concentrations, for each of the three pre-

157 treatments. The first pre-treatment, "Freezing", was chosen to represent the effects of repeated freeze-

158 thaw cycles that some Malaise trap samples may be experiencing during long-term storage. Specifically,

159 we subjected our samples to three cycles of $16 \mathrm{~h}$ at $-20^{\circ} \mathrm{C}$ and $8 \mathrm{~h}$ at room temperature. The second pre-

160 treatment, "Drying", tests the effects of specimen drying, included in some non-destructive DNA

161 extraction protocols (e.g., Nielsen, Gilbert, Pape, \& Bohmann, 2019, Vesterinen et al., 2016) prior to

162 digestion in the lysis buffer. Samples may also dry up accidentally, especially in difficult field conditions.

163 In this treatment, we carefully poured away the ethanol from the tube, dried the insects for $24 \mathrm{~h}$ in the

164 tube at room temperature, and afterwards, added $40 \mathrm{~mL}$ of ethanol at the original concentration. The last

165 treatment was a control: the communities remained in ethanol at room temperature. After the pre-

166 treatment, all tubes were manually vortexed in a horizontal position for $1 \mathrm{~min}$, corresponding to the

167 "Gentle" treatment described in Experiment 1. The damage was assessed and scored as in Experiments 1

168 and 2.

169

170 Experiment 4: Effect of ethanol concentration on DNA preservation

Peer) reviewing PDF | (2020:11:55112:1:0:NEW 23 Dec 2020) 
171 In the last experiment, we used insect samples from the first experiment to test the effects of ethanol

172 concentration on DNA preservation (Figure 1E). After the brittleness assessment, the insects were placed

173 back in the tubes with their corresponding ethanol concentration and stored at room temperature, in

174 shaded boxes, for twelve months. Then, for each of the seven experimental species preserved in eight

175 ethanol concentrations (30, 50, 70, 80, 90, 95, 97, and 99\%), we took a single insect specimen (Dacnusa)

176 or a single insect leg (other species) from each of the five tubes from the "Gentle" treatment. We reasoned

177 that in specimens stored at lower ethanol concentrations after extended storage, DNA is likely to be more

178 degraded, containing fewer amplifiable copies of target genes in the same amount of insect tissue. To test

179 this, we quantified differences in PCR template amounts across ethanol concentrations by adding the

180 same amount of an artificial target to each sample of a given species prior to DNA extraction.

181 Subsequently, we used the extracted DNA to prepare cytochrome oxidase I (COI) amplicon libraries that

182 were then sequenced and assessed the ratios of an artificial target to insect COI gene in the resulting 183 amplicon datasets.

184

185

Amplicon library preparation and bioinformatic processing (Experiment 4)

186 Each sample (one entire specimen for Dacnusa or a single leg for the rest of species) was placed in a 2 $187 \mathrm{~mL}$ tube, containing $190 \mu \mathrm{L}$ lysis buffer $(0.4 \mathrm{M} \mathrm{NaCl}, 10 \mathrm{mM}$ Tris-HCl, $2 \mathrm{mM}$ EDTA, $2 \% \mathrm{SDS}), 10 \mu \mathrm{L}$ of 188 proteinase $\mathrm{K}$ and ceramic beads $(2.8 \mathrm{~mm}$ and $0.5 \mathrm{~mm})$. Samples were homogenized on an Omni Bead 189 Ruptor 24 homogenizer during two $30 \mathrm{~s}$ cycles at the speed of $5 \mathrm{~m} / \mathrm{s}$ and then incubated at $56^{\circ} \mathrm{C}$ for 2 190 hours. From this homogenate, $40 \mu \mathrm{L}$ (corresponding to $20 \%$ of the total lysate volume) was mixed with a 191 predefined amount of a linearized spike-in plasmid carrying an artificial COI target. The plasmid 192 contained a 471-base insert developed based on the Calliphora vomitoria barcode region sequence, where 193 outside of the conserved primer regions, approximately $40 \%$ of bases were substituted. Plasmid 194 quantities were determined for each species during pilot experiments and ranged from 11,000 to 317,000 195 copies per sample (Supplementary Material, Table S7). DNA was purified by mixing $42 \mu \mathrm{L}$ of each 196 sample ( $40 \mu \mathrm{L}$ of homogenate and $2 \mu \mathrm{L}$ of plasmid) with $80 \mu \mathrm{L}$ of home-made Sera-mag SpeedBead 
197 solution, separating the beads on a magnetic plate, washing with $80 \%$ ethanol twice, and finally, 198 resuspending the DNA in $20 \mu \mathrm{L}$ of TE buffer.

199 COI amplicon libraries for 278 samples, plus six controls (two for lysis buffer, two for PCR, and two 200 for indexing), were prepared following a two-step PCR library preparation protocol (Method 4, Glenn et 201 al., 2011). In the first PCR, the 458-bp target region was amplified using template-specific primers with 202 variable-length inserts and Illumina adapter tails. After the bead-purification of products, products were 203 completed with Illumina adapters unique for each library in the second, indexing PCR. The 204 oligonucleotide sequences and reaction conditions are provided in Supplementary Material S5. The 205 pooled libraries were sequenced in a multiplexed Illumina MiSeq v3 lane (2 x $300 \mathrm{bp}$ reads) at the 206 Institute of Environmental Sciences of Jagiellonian University (Kraków, Poland).

207 The amplicon data was processed using Mothur v1.44.2 (Schloss et al., 2009). Reads were merged into 208 contigs, had primers trimmed, were dereplicated, and singleton genotypes were removed. These filtered 209 data were then used for $97 \%$ OTU picking. The commands used are provided in the Supplementary 210 Material. Next, the dominant OTUs corresponding to target insects and the artificial target were 211 identified, and for all samples, the insect OTU to artificial target OTU ratio was calculated. Additionally, 212 because for three species (Drosophila, Formica, and Macrolophus) additional COI OTUs (likely 213 corresponding to nuclear mitochondrial pseudogenes) were relatively abundant, we repeated the analyses 214 after summing up the reads of all OTUs that were at least $90 \%$ identical to the dominant sequence. We 215 then translated the abundance ratios to an estimated number of insect COI copies in each sample and 216 standardized the values relative to the median value for $95 \%$ ethanol concentration for that same species.

\section{Statistical analyses}

219 All analyses were conducted in $\mathrm{R}$ ( $\mathrm{R}$ Core Team, 2017) using the base package and the packages 220 'glmmTMB' (Brooks et al., 2017), 'DHARMa' (Hartig, 2019), and 'emmeans' (Lenth, 2019). 221 Visualizations were generated with package 'ggplot2' (Wickham, 2016). For the morphological 222 preservation analysis, all types of appendages were considered together $($ Appendages $=$ Legs + Wings + 
223 Antennae + Head), and each species was analyzed separately for each experiment. If the head was lost, 224 the antennae were also scored as lost. A generalized linear mixed effects model was fitted to the data with 225 the number of appendages lost as a function of Treatment and Concentration, and their interaction, as 226 fixed effects, and Tube (= replicate) as a random effect, assuming a log-link and Poisson distribution 227 (function $g \operatorname{lmm} T M B$ from package glmmTMB). As some species did not lose any appendages at all in 228 certain treatments, zero inflation in the model was tested by simulating scaled residuals with the function 229 simulateResiduals (package DHARMa) with the model without considering zero inflation, and checking 230 the presence of zero inflation (function testZeroInflation from package DHARMa). If significant, a term 231 for controlling zero inflation dependent on Concentration was included in the model. Subsequently, an 232 analysis of variance (ANOVA) was used to determine whether Treatment, Concentration, and their 233

234 235

239

240

241

242

243

244 245

246

\section{Results}

248 Experiment 1: Effects of ethanol concentration on specimen brittleness 
249 For most insect species tested, we found a significant effect of ethanol concentration on the number of 250 broken or lost appendages, but the nature of the effect differed among species (Figure 2). In general, the 251 number of broken appendages rose with the concentration of preservative ethanol. However, the 252 magnitude of the effect varied dramatically, and only in some cases, it was significant. The lowest ethanol 253 concentrations (30 or $50 \%$ ) were also associated with an increased loss of appendages compared to 254 intermediate concentrations. However, the difference between our "Gentle" and "Vigorous" treatments, 255 with a two-fold difference in vortexing time, was relatively small.

256 When fitted to the model, ethanol concentration alone had a very significant effect on the number of 257 lost appendages in Macrolophus, Aphidoletes, and Calliphora but not in Drosophila, Dacnusa or 258 Dermestes (Formica did not lose enough appendages to fit a model; Table 1, Table S2). In both 259 Aphidoletes and Calliphora, intermediate concentrations of ethanol were optimal for the preservation of 260 appendages, whereas the pattern in Macrolophus was less clear. In Aphidoletes and Drosophila, the 261 shaking regime, as well as the interaction of concentration and shaking type, also had a significant effect

262

263

264

265

266

267 268 269 270 271

\section{Experiment 2: Effects of transport regimes}

273 In the transport experiment, only three species produced enough data points to fit a model: Macrolophus, 274 Aphidoletes, and Drosophila (Figure 3, Table 2, Table S3). For Drosophila, no significant differences 
275 were found between any transport treatment, ethanol concentration, or their interaction. This is consistent 276 with the results from Experiment 1, showing that Drosophila is quite robust to the two different shaking 277 regimes we tried under a broad range of ethanol concentrations, including the two concentrations (70\% 278 and $95 \%$ ) we used in the transport experiment.

279 For the remaining two species, Macrolophus and Aphidoletes, specimens carried by a careful 280 (Walking) technician sustained less damage than those carried by a reckless (Running) technician or sent 281 by mail (Postnord). When the samples were treated with care (Walking), preservative ethanol 282 concentration did not affect the number of appendages lost by either species. However, in those treated 283 with less care, including Running and Postnord treatments, both species sustained significantly less 284 damage when preserved in 70\% ethanol.

285

286

Experiment 3: Effects of freeze-thaw cycles and drying

287

288

289

290

291

292

293

294 295 296 297 298 299

As in the previous cases, the responses to freezing/drying treatments varied among species (Figure 4, Table 3, Table S4). The three toughest species (Dacnusa, Formica, and Dermestes) had to be excluded from the analyses, as they did not generate enough data to fit a model. In the remaining species, we did not observe a significant treatment effect, that is, drying the specimens or exposing them to repeated cycles of freezing and thawing did not seem to affect their brittleness (Figure 4). The only exception was Macrolophus, where we observed a mild positive effect of drying the specimens. Regardless of treatment, Aphidoletes, Drosophila, and Calliphora lost more appendages on average when stored at $95 \%$, although the effect was only significant in Aphidoletes. In Macrolophus, there was no clear difference between the brittleness of specimens stored at $70 \%$ and at $95 \%$. These species-specific responses to different ethanol concentrations closely match those seen in Experiment 1 (Fig. 2).

\section{Experiment 4: Effect of ethanol concentration on DNA preservation}

For the 284 experimental libraries (278 insects and 6 blanks), we obtained a total of $6,061,251$ read pairs. Of these, 5,444,2555 reads, for 276 insect libraries (range 948 - 196,774, median 17,180 per sample), 
301 passed all analysis stages and were used for abundance comparisons (Table S7). Across libraries, the

302 median abundance of the artificial target OTU relative to the dominant OTU for the insect species was 3030.136 (range $0.003-614$ ); the value dropped slightly when all OTUs with $>90 \%$ identity to the 304 reference sequence were combined (0.134). Negative PCR and indexing controls had almost no COI reads $305(0-18)$, and in the DNA extraction controls, most reads $(1760-8610,>98.5 \%$ of the total $)$ 306 corresponded to the spike-in plasmid.

307 In all studied species, we found significant differences in the estimated COI target copy number 308 between ethanol concentrations (Table S8) (Fig. 5). A gradual decrease in the number of COI copies with 309 decreasing ethanol concentration is visible in all species, although the strength of the effect and the 310 concentration at which it sets in varies (Fig. 5). Post-hoc analyses revealed a significant decrease in target 311 copy numbers between at least some of the lower-concentration treatments and the $95 \%$ ethanol 312 reference in all species. Specifically, the target copy number was significantly lower than in the 95\% 313 reference in samples preserved in $30 \%$ ethanol (7 out of 7 species), $50 \%$ (7 species), $70 \%$ (5 species), $31480 \%$ (3 species) and in one of the species preserved at $90 \%$ (Macrolophus). Samples preserved in $97 \%$ 315 and $99 \%$ ethanol did not differ significantly from the $95 \%$ reference. The magnitude of the effect of the 316 ethanol concentration varied among species: for example, in samples preserved at the lowest ethanol 317 concentrations (30 and $50 \%$ ), the decrease in the number of COI copies relative to the $95 \%$ reference 318 ranged from 3 to 440 -fold.

319 Results obtained from a dataset with all OTUs at $>90 \%$ identity to reference sequence for that species considered do not differ qualitatively from the ones summarized above (Table S8).

\section{Discussion}

324 It is surprising that there are so few quantitative studies of the effect of ethanol concentration on the 325 preservation of insects for morphological and molecular study. One possible reason for this is the 
326

327

328

329

330

331

332

333

334

335

336

337

338

339

340

341

342

343

344

345

346

347

348

349

350

351

difficulty of quantifying morphological preservation, but also the preservation of DNA for diverse sequencing-based applications. Our approaches allowed us to address both of these challenges.

The criterion we used here to evaluate morphological preservation, the number of lost appendages, has the major advantage that it is fast and easy to measure. However, it captures only one aspect of morphological preservation, namely brittleness. Brittleness is important in many contexts, for instance, when handling, examining or mounting specimens, but it is not an ideal measure of the preservation of fine morphological details, or the status of internal anatomy. For instance, we noted a clear discrepancy between brittleness and morphological preservation with the drying pre-treatment. Nevertheless, we believe our approach is a good starting point for further enquiry into the effect of ethanol concentration on the preservation of insects. Undoubtedly, it would be valuable to keep exploring more sophisticated measures of morphological conservation.

To assess DNA preservation, we used a quick measure of one simple aspect of DNA degradation: the absolute abundance of a certain 458-base long DNA fragment, part of the mitochondrial genome. It is clearly not a perfect measure of the overall DNA preservation. However, as DNA degradation happens primarily by the accumulation of random breaks within the DNA strands (although other processes play a role, for example, deamination of cytosines), it is unlikely that many longer fragments would remain once the abundance of 458-base targets decreases significantly. Thus, our results are of direct relevance to sequencing approaches that do not require high-molecular-weight DNA, but also informative to researchers that sequence long DNA fragments (e.g., PacBio or Oxford Nanopore sequencing).

The use of spike-in plasmids with artificial targets in amplicon sequencing experiments has been gaining popularity as a method for the quantification of microbiomes (Tkacz, Hortala \& Poole, 2018; Tourlousse et al., 2017), while spike-in standards consisting of sequences of actual species have been used for abundance estimation in eukaryotic metagenomic studies (Ji et al., 2020). However, to our knowledge, they have not been used to assess DNA degradation. We found the absolute quantification of COI targets using spike-ins to be a quick, inexpensive, and, in our opinion, accurate and biologically informative way of gathering DNA preservation data.

Peer) reviewing PDF | (2020:11:55112:1:0:NEW 23 Dec 2020) 

an impact on the fragility of insect specimens, even though the effects vary among taxonomic groups.

354 High concentrations tend to increase brittleness, especially in weakly sclerotized insects. We also noted a 355 tendency of more disruptive treatments to have a stronger effect on specimens preserved in high ethanol 356 concentrations. Regarding DNA preservation, the expectations are also met, as we observed a lower 357 proportion of suitable PCR-template DNA fragments in the DNA extracts of those individuals stored at 358 lower ethanol concentrations after only one year.

359 The effect of ethanol concentrations on morphological preservation was most pronounced in the most 360 weakly sclerotized species (Aphidoletes and Calliphora in particular). As the connective and muscular 361 tissues are dried and made brittle by high ethanol concentrations, it is reasonable that insects with less 362 exoskeletal anchorage in their joints will be more severely affected. However, interestingly, the negative 363 effect of low ethanol concentrations was noticeable also in Dermestes. These beetles were very robust to damage at high ethanol concentrations, but tended to lose elytra - abdomen and head too - at low concentrations. The effect was not significant, but likely primarily because of lower numbers of suspect that this effect would have been significant. It would be interesting to test whether the increased penetration into tissues, or perhaps decomposing microorganisms likely harbored by these carcass-feeding beetles (these beetles were obtained from the dermestarium at the Swedish Museum of Natural History, 371 where they clean off the soft tissue of vertebrate carcasses before the skeletal parts are included in the 372 collections).

373 Our transport experiment clearly shows that the concentration of preservative ethanol has a strong 374 effect on how fragile insect specimens are. Fragile specimens survive transport much better if stored in 70 $375 \%$ ethanol than if stored in $95 \%$ ethanol (Figure 3). The experiment also demonstrates that it is important 376 to handle samples carefully. A careless technician can cause considerably more damage than shipping the 377 specimens more than $400 \mathrm{~km}$ by a much-criticized national mail service. It should be noted, however, that 
378 all tubes were filled with liquid to nearly maximum capacity before being shipped, as generally 379 recommended for safe transport (Martin, 1977). The results might have been different if the tubes had 380 been half-empty.

381 Subjecting preserved specimens to repeated freezing and thawing cycles did not significantly affect 382 their tendency to lose appendages. Similarly, drying the specimens and then reimmersing them in ethanol 383 had no noticeable negative effect. In fact, drying had a slight positive effect on Macrolophus specimens, 384 which lost fewer appendages after drying than in the control treatment. However, these results do not 385 mean that the morphology is preserved intact through these treatments. For instance, we observed that 386 many individuals from most species that had been dried had shrunken heads and abdomens that could 387 complicate their taxonomic examination. It seems likely that also internal anatomy was affected both by 388 the freezing-thawing cycles and by the drying-reimmersion treatment. Thus, it would be valuable to 389 reinvestigate the effects of these treatments using other criteria for morphological preservation than the simple measure of appendage loss that we used.

As remarked previously, the effect of ethanol concentration varied strikingly among the different insect species we examined. Although our sample size is small, some results may indicate patterns that apply more broadly to particular taxonomic groups of insects. For instance, it is notable that both hymenopterans we examined, Dacnusa and Formica, were quite robust regardless of treatment, despite the fact that Dacnusa is a small species of relatively delicate build. These results seem to be in concordance with previous studies that show that hymenopteran bodies resist the entrance of ethanol to 397 the point that sometimes small holes must be carved in their exoskeleton to allow DNA preservation 398 (Dillon, Austin \& Bartowsky, 1996; Mandrioli, 2008). However, long-term storage in absolute ethanol 399 can nevertheless make ants brittle (King \& Porter, 2004). The three dipterans we examined (Drosophila, 400 Calliphora and Aphidoletes) were all quite sensitive to the ethanol concentration, regardless of striking 401 differences in size, body shape, and level of sclerotization. They were also consistently among the insects 402 most affected by the different treatments we exposed them to. Coleoptera was represented by only one 403 taxon in our study (Dermestes), but beetles are generally more sclerotized than other insects, and it is no 
404 surprise that Dermestes belonged to the most robust of the insects we studied. Hemiptera were similarly 405 represented by a single taxon in our study (Macrolophus). This species was among the most fragile we 406 studied, but the order does present a wide variety of body types, and future studies will have to show to 407 what extent Macrolophus is representative.

408 In general, the opposite pattern to the preservation of morphology was observed for the preservation of 409 the DNA: the higher the ethanol concentration, the better preserved the DNA was. For most species, 410 individuals preserved at concentrations of $80 \%$ ethanol or lower produced significantly fewer copies of 411 the targeted PCR fragment than those preserved at $95 \%$. This shows that, even when an amplification 412 product can be obtained through PCR, there is, indeed, DNA degradation at these ethanol concentrations. 413 Interestingly, concentrations of 97 and $99 \%$ ethanol never differ significantly from $95 \%$, albeit in some 414 species like Dermestes the average number of COI template copies was visibly higher at both of these concentrations. The concentration at which significant degradation of DNA was first observed varied among species. For four of the seven species, the preservation of the DNA was significantly worse from $41770-80 \%$ and down, while for Macrolophus, even a concentration as high as $90 \%$ underperformed 418 compared to $95 \%$. On the other hand, the DNA of Dacnusa and Calliphora was not significantly 419 degraded until the concentration was lowered to 50 or $30 \%$. The mechanisms underlying these differences are unknown to us. One of the possible explanations offered above for the good morphological preservation of hymenopterans (namely, the resistance of their bodies to the entrance of ethanol) is directly at odds with the good preservation of DNA (extremely good in Dacnusa, quite good also in Formica). This suggests that the morphological solidity of hymenopterans is due to other causes.

424 For instance, the body is more distinctly divided into hard sclerites in Hymenoptera than is the case for 425 many other insect orders, like Diptera. Potentially, this could be associated with an decreased tendency to 426 lose appendages.

427 Regardless of the causes, the variation among taxa in the effects of ethanol concentration on DNA 428 preservation is highly relevant for metabarcoding studies. Metabarcoding involves the amplification of a 429 barcoding gene sequence from DNA templates of many species simultaneously, so differences in the 
430 preservation of DNA among taxa could potentially result in significant biases, with the poorly preserved 431 species contributing less than others to the pool of accessible template DNA copies. Minimally, this will 432 cause problems with abundance estimation from metabarcoding data; in the worst case, poorly preserved 433 species might go completely undetected, especially if they are rare.

434 Our study may be the first systematic study of the effect of ethanol concentration on morphological 435 and molecular preservation of insects. Our results largely confirm the commonly held belief that 436 intermediate concentrations of ethanol, around $70-80 \%$, are generally the best for morphological 437 preservation, as high concentrations (above $90 \%$ ) tend to make specimens fragile, whereas lower 438 concentration in many cases allow some level of degradation. In contrast, the optimal DNA preservation 439 is achieved at ethanol concentrations of $>90 \%$. Unfortunately this means that there is a conflict between 440 preserving insects for morphological and for molecular work, as the ethanol concentrations that are ideal 441 for the former purposes result in higher degradation rates of DNA. Is it possible to find a treatment that is 442 ideal for both purposes? Stein et al. (2013) showed that if initial preservation is made in $95 \%$ ethanol, 443 good PCR results can be obtained even after storing the insects in $70 \%$ ethanol for an extended period of 444 time. However, this is the exact contrary of what is recommended for morphological study, where a 445 gradual increase in the ethanol concentration is recommended to avoid fast desiccation of the tissues. 446 However, whether initial preservation in $95 \%$ and subsequent transfer to $70 \%$ for storage reduces the 447 brittleness is unknown. Recently, researchers have directed their attention towards preservatives based on 448 propylene glycol (Nakamura, Tamura, Taki \& Shoda-Kagaya, 2020; Moreau, Wray, Czekanski-Moir \& 449 Rubin, 2012; Robinson, Porter, Wright \& Hajibabaei, 2020). Lower flammability, evaporation, and cost 450 make propylene glycol an attractive alternative to ethanol for trapping, transporting, and storing insect 451 samples. Nevertheless, although these results look promising, more studies are needed to determine the 452 quality of DNA and morphological preservation for different insect groups, under a range of conditions, 453 for these preservatives.

454 For now, we do not have a preservation regimen that is ideal for both morphology and molecules. This 455 must be taken into account when planning large collecting campaigns that aim to preserve material for 
456 both morphological and molecular work. For instance, we note that catches from Malaise traps, which are

457 frequently used in insect inventories, contain a large portion of Diptera specimens (Hebert et al., 2016;

458 Karlsson et al., 2020; Ronquist et al., 2020), a group whose morphology is heavily affected by high 459 ethanol concentrations. Hymenoptera, on the other hand, is abundant as well in Malaise traps, and not as 460 prone to morphological damage. These differences need to be considered at the stage of experimental 461 design: the relative importance of morphological against DNA data, or perhaps the focus on certain 462 taxonomic groups, should guide the selection of preservative composition and preservation conditions. In 463 any case, if Malaise trap inventory projects intend to preserve material for molecular and morphological 464 study, it may be necessary to store the material at ethanol concentrations that are not ideal neither for 465 molecular nor for morphological work.

466

467 Acknowledgements

468 We are thankful to Dave Karlsson from Station Liné for receiving and returning the parcels with the tubes 469 of the PostNord treatment. This project was supported by the European Union's Horizon 2020 research 470 and innovation programme under the Marie Skłodowska-Curie grant agreement no. 642241 (BIG4 471 project, https://big4-project.eu), by the Knut and Alice Wallenberg Foundation (KAW 2017.088), Polish 472 National Agency for Academic Exchange grant PPN/PPO/2018/1/00015 and Polish National Science 473 Centre grant 2018/31/B/NZ8/01158. Ela Iwaszkiewisz contributed to the design of the artificial target for 474 DNA preservation assessment, and Anna Michalik and Monika Prus prepared the plasmid for use. We 475 acknowledge PhyloPic platform (http://phylopic.org) for the silhouettes of the insects in Figure 1, as well 476 as the authors of the silhouette corresponding to Dacnusa sibirica (Maxime Dahirel (digitisation), Kees 477 van Achterberg et al. (doi: 10.3897/BDJ.8.e49017)(original publication), CC BY 3.0) and to Macrolophus 478 pygmaeus (Dave Angelini, CC BY 3.0).

479

480 Authors' Contributions 
481 DM, FR and PŁ conceived and designed the study; DM, MB and PŁ prepared the communities and

482 conducted the experiment, collected and analysed the data, and prepared the figures; DM wrote the first 483 draft of the manuscript. All authors contributed critically to subsequent versions of the manuscript and 484 gave final approval for publication.

485

486 Data Availability

487 All raw appendages counts, sequence reads counts and provenance of the insects used can be found in the 488 tables of the supplementary material of this article. The amplicon sequencing data was deposited in NCBI 489 Short Read Archive (BioProject PRJNA662328).

490

491

492

\section{References}

493

494

495

496

497

498

499

500

501

502

503

504

505

506

507

Baird, D. J., Pascoe, T. J., Zhou, X. \& Hajibabaei, M. (2011). Building freshwater macroinvertebrate DNA-barcode libraries from reference collection material: formalin preservation vs specimen age. Journal of the North American Benthological Society, 30(1), 125-130.

Boyle, R. (1664). Some considerations touching the vsefulnesse of experimental naturall philosophy. London: Henry Hall for Richard Davis.

Brooks, M. E., Kristensen, K., van Benthem, K. J., Magnusson, A., Berg, C. W., Nielsen, A., ... \& Bolker, B. M. (2017). glmmTMB balances speed and flexibility among packages for zero-inflated generalized linear mixed modeling. The R journal, 9(2), 378-400.

Carew, M. E., Metzeling, L., St Clair, R. \& Hoffmann, A. A. (2017). Detecting invertebrate species in archived collections using next-generation sequencing. Molecular Ecology Resources, 17(5), 915930.

Carew, M. E., Coleman, R. A. \& Hoffmann, A. A. (2018). Can non-destructive DNA extraction of bulk invertebrate samples be used for metabarcoding? PeerJ, 6(1702), e4980.

Dillon, N., Austin, A. D. \& Bartowsky, E. (1996). Comparison of preservation techniques for DNA extraction from hymenopterous insects. Insect Molecular Biology, 5(1), 21-24. 
508 Glenn TC, Pierson TW, Bayona-Vásquez NJ, Kieran TJ, Hoffberg SL, Thomas IV JC, ... \& Faircloth BC.

509

510

511

512

513

514

515

516

517

518

519

520

521

522

523

524

525

526

527

528

529

530

531

532

533

534

535 (2019). Adapterama II: universal amplicon sequencing on Illumina platforms (TaggiMatrix) PeerJ, 7, e7786.

Hartig, F. (2019). DHARMa: residual diagnostics for hierarchical (multi-level/mixed) regression models. R package.

Hebert, P. D. N., Ratnasingham, S., Zakharov, E. V., Telfer, A. C., Levesque-Beaudin, V., Milton, M. A., ... \& deWaard, J. R. (2016). Counting animal species with DNA barcodes: Canadian insects. Philosophical Transactions of the Royal Society of London. Series B, Biological Sciences, $371(1702), 20150333$.

Janzen, D. H., Hallwachs, W., Blandin, P., Burns, J. M., Cadiou, J.-M., Chacon, I., .. \& Wilson, J. J. (2009). Integration of DNA barcoding into an ongoing inventory of complex tropical biodiversity. Molecular Ecology Resources, 9(s1), 1-26.

Ji, Y., Huotari, T., Roslin, T., Schmidt, N. M., Wang, J., Yu, D. W., \& Ovaskainen, O. (2020). SPIKEPIPE: A metagenomic pipeline for the accurate quantification of eukaryotic species occurrences and intraspecific abundance change using DNA barcodes or mitogenomes. Molecular Ecology Resources, 20(1), 256-267.

King, J. R. \& Porter, S. D. (2004). Recommendations on the use of alcohols for preservation of ant specimens (Hymenoptera, Formicidae). Insectes Sociaux, 51(2), 197-202.

Karlsson, D., Hartop, E., Forshage, M., Jaschhof, M., \& Ronquist, F. (2020). The Swedish Malaise Trap Project: A 15 Year Retrospective on a Countrywide Insect Inventory. Biodiversity Data Journal, 8, e47255.

Lenth, R. (2018). Emmeans: Estimated marginal means. Aka Least-squares Means, R. https://CRAN.Rproject.org/package $=$ emmeans

von Linné, C. (1764). Museum Sae Rae Mtis Ludovicae Ulricae reginae. Holmiae: Salvius.

Mandrioli, M. (2008). Insect collections and DNA analyses: how to manage collections? Museum Management and Curatorship, 23(2), 193-199.

Martin, J. E. H. (1977). The insects and arachnids of Canada. Part 1: Collecting, preparing, and preserving insects, mites, and spiders. Hull: Publication 1643, Research Branch, Canada Department 
536

537

538

539

540

541

542

543

544

545

546

547

548

549

550

551

552

553

554

555

556

557

558

559

560

561

562

563

of Agriculture.

Matos-Maraví, P., Duarte Ritter, C., Barnes, C. J., Nielsen, M., Olsson, U., Wahlberg, N., ... \& Antonelli, A. (2019). Biodiversity seen through the perspective of insects: 10 simple rules on methodological choices and experimental design for genomic studies. PeerJ, 7, e6727.

Moreau, C. S., Wray, B. D., Czekanski-Moir, J. E., \& Rubin, B. E. (2013). DNA preservation: a test of commonly used preservatives for insects. Invertebrate Systematics, 27(1), 81-86.

Nagy, Z. T. (2010). A hands-on overview of tissue preservation methods for molecular genetic analyses. Organisms Diversity \& Evolution, 10(1), 91-105.

Nakamura, S., Tamura, S., Taki, H., \& Shoda-Kagaya, E. (2020). Propylene glycol: a promising preservative for insects, comparable to ethanol, from trapping to DNA analysis. Entomologia Experimentalis et Applicata, 168(2), 158-165.

Nielsen, M., Gilbert, M. T. P., Pape, T., \& Bohmann, K. (2019). A simplified DNA extraction protocol for unsorted bulk arthropod samples that maintains exoskeletal integrity. Environmental DNA, edn3.16-11.

R Development Core Team (2017). R: A language and environment for statistical computing. Vienna, Austria: R Foundation for Statistical Computing

Robinson, C. V., Porter, T. M., Wright, M. T., \& Hajibabaei, M. (2020). Propylene glycol-based antifreeze as an effective preservative for DNA metabarcoding of benthic arthropods. bioRxiv, 2020.02.28.970475.

Ronquist, F., Forshage, M., Häggqvist, S., Karlsson, D., Hovmöller, R., Bergsten, J., .. \& Gärdenfors, U. (2020). Completing Linnaeus's inventory of the Swedish insect fauna: Only 5000 species left? PLos One, 15(3), e0228561.

Schloss, P. D., Westcott, S. L., Ryabin, T., Hall, J. R., Hartmann, M., Hollister, E. B., ... \& Sahl, J. W. (2009). Introducing mothur: open-source, platform-independent, community-supported software for describing and comparing microbial communities. Applied and Environmental Microbiology, 75(23), 7537-7541.

Shokralla, S., Gibson, J. F., Nikbakht, H., Janzen, D. H., Hallwachs, W. \& Hajibabaei, M. (2014). Nextgeneration DNA barcoding: using next-generation sequencing to enhance and accelerate DNA 
564

565

566

567

568

569

570

571

572

573

574

575

576

577

578

579

580

581

582

583

584

585

586

587

588

589

590

591

592

593

594

595

Srinivasan, M., Sedmak, D. \& Jewell, S. (2002). Effect of fixatives and tissue processing on the content and integrity of nucleic acids. The American Journal of Pathology, 161(6), 1961-1971.

Stein, E. D., White, B. P., Mazor, R. D., Miller, P. E. \& Pilgrim, E. M. (2013). Evaluating Ethanol-based Sample Preservation to Facilitate Use of DNA Barcoding in Routine Freshwater Biomonitoring Programs Using Benthic Macroinvertebrates. PLoS One, 8(1), e51273.

Tkacz, A., Hortala, M., \& Poole, P. S. (2018). Absolute quantitation of microbiota abundance in environmental samples. Microbiome, 6(1), 1-13.

Tourlousse, D. M., Yoshiike, S., Ohashi, A., Matsukura, S., Noda, N., \& Sekiguchi, Y. (2017). Synthetic spike-in standards for high-throughput 16S rRNA gene amplicon sequencing. Nucleic Acids Research, 45(4), e23.

Vesterinen, E. J., Ruokolainen, L., Wahlberg, N., Peña, C., Roslin, T., Laine, V. N., ... \& Lilley, T. M. (2016). What you need is what you eat? Prey selection by the bat Myotis daubentonii. Molecular Ecology, 25(7), 1581-1594.

Wickham, H. (2016). ggplot2: elegant graphics for data analysis. Springer.

\section{Supplementary Material}

Supplementary material, including the raw counts of lost appendages, can be found in the electronic version of the article. 
599 Table 1 Effect of Concentration, treatment and their interaction in the number of lost appendages for each 600 species in Experiment 1. F. rufa did not produce enough data points to fit a model. Shown are Type III 601 test of fixed effects if the model described. Asterisks indicate levels of significance: * p-value $<0.05 ; * *$ 602 p-value $<0.01 ; * * *$ p-value $<0.001$.

603

\begin{tabular}{|c|c|c|c|}
\hline Effect & $\chi^{2}$ & $\overline{\text { Df }}$ & p-value \\
\hline \multicolumn{4}{|l|}{ Macrolophus pygmaeus } \\
\hline Concentration & 35.0614 & 7 & $0.0001 * * *$ \\
\hline Treatment & 1.7642 & 1 & 0.1841 \\
\hline Concentration:Treatment & 12.4535 & 7 & 0.0866 \\
\hline \multicolumn{4}{|l|}{ Aphidoletes aphidimyza } \\
\hline Concentration & 170.2500 & 7 & $0.0001 * * *$ \\
\hline Treatment & 4.0480 & 1 & $0.0442 *$ \\
\hline Concentration:Treatment & 18.350 & 7 & $0.0104 *$ \\
\hline \multicolumn{4}{|l|}{ Drosophila hydei } \\
\hline Concentration & 10.1877 & 7 & 0.1781 \\
\hline Treatment & 5.3045 & 1 & $0.0212 *$ \\
\hline Concentration:Treatment & 15.7197 & 7 & $0.0278 *$ \\
\hline \multicolumn{4}{|l|}{ Dacnusa sibirica } \\
\hline Concentration & 4.3189 & 7 & 0.7424 \\
\hline Treatment & 0.0000 & 1 & 1.0000 \\
\hline Concentration:Treatment & 3.9310 & 7 & 0.7877 \\
\hline \multicolumn{4}{|l|}{ Calliphora vomitoria } \\
\hline Concentration & 24.5232 & 7 & $0.0009 * * *$ \\
\hline Treatment & 0.6392 & 1 & 0.4239 \\
\hline Concentration:Treatment & 2.3005 & 7 & 0.9413 \\
\hline \multicolumn{4}{|l|}{ Dermestes haemorrhoidalis } \\
\hline Concentration & 11.5213 & 7 & 0.1174 \\
\hline Treatment & 0.3203 & 1 & 0.5714 \\
\hline Concentration:Treatment & 1.6935 & 7 & 0.9748 \\
\hline
\end{tabular}


607

608

609

610

611

612 Table 2 Effect of Concentration, treatment and their interaction in the number of lost appendages for each

613 species in Experiment 2. Only M. pygmaeus, A. aphidimyza and D. hydei produced enough data points to

614 fit the model. Shown are Type III test of fixed effects if the model described. Asterisks indicate levels of

615 significance: $*$ p-value $<0.05 ; * *$ p-value $<0.01 ; * *$ p-value $<0.001$.

616

\begin{tabular}{lrrr}
\hline Effect & \multicolumn{1}{c}{$\chi^{2}$} & Df & \multicolumn{1}{c}{ p-value } \\
\hline Macrolophus pygmaeus & & & \\
Concentration & 0.0000 & 1 & 1.0000 \\
Treatment & 19.3886 & 2 & $0.0006^{* * *}$ \\
Concentration:Treatment & 0.8273 & 2 & 0.6612 \\
Aphidoletes aphidimyza & & & \\
Concentration & 0.5238 & 1 & 0.4692 \\
Treatment & 11.5596 & 2 & $0.0031 * *$ \\
Concentration:Treatment & 21.5104 & 2 & $0.0002 * * *$ \\
Drosophila hydei & & & \\
Concentration & 0.9152 & 1 & 0.3387 \\
Treatment & 3.0386 & 2 & 0.2189 \\
Concentration:Treatment & 1.6271 & 2 & 0.4433 \\
\hline
\end{tabular}

617

618

619

620

621

622

623

624

625

626

627

628 
636 Table 3 Effect of Concentration, treatment and their interaction in the number of lost appendages for each 637 species in Experiment 3. D. haemorrhoidalis, F. rufa and D. sibirica did not produced enough data points 638 to fit the model. Shown are Type III test of fixed effects if the model described. Asterisks indicate levels 639 of significance: * p-value $<0.05 ; * *$ p-value $<0.01 ; * *$ p-value $<0.001$.

640

\begin{tabular}{lrrr}
\hline Effect & \multicolumn{1}{c}{$\chi^{2}$} & Df & \multicolumn{1}{c}{ p-value } \\
\hline Macrolophus pygmaeus & & & \\
Concentration & 0.1984 & 1 & 0.6560 \\
Treatment & 21.4882 & 2 & $0.0002 * * *$ \\
Concentration:Treatment & 8.5936 & 2 & $0.0136 *$ \\
Aphidoletes aphidimyza & & & \\
Concentration & 40.8062 & 1 & $0.0001 * * *$ \\
Treatment & 1.1408 & 2 & 0.5653 \\
Concentration:Treatment & 1.5768 & 2 & 0.4546 \\
Drosophila hydei & & & \\
Concentration & 0.6375 & 1 & 0.4246 \\
Treatment & 6.0051 & 2 & $0.0496 *$ \\
Concentration:Treatment & 6.3404 & 2 & $0.0419 *$ \\
Calliphora vomitoria & & & \\
Concentration & 0.1544 & 1 & 0.6943 \\
Treatment & 4.5955 & 2 & 0.1005 \\
Concentration:Treatment & 6.1628 & 2 & $0.0459 *$ \\
\hline & & &
\end{tabular}


647

648

649

650

651

652

653

654

655

656 


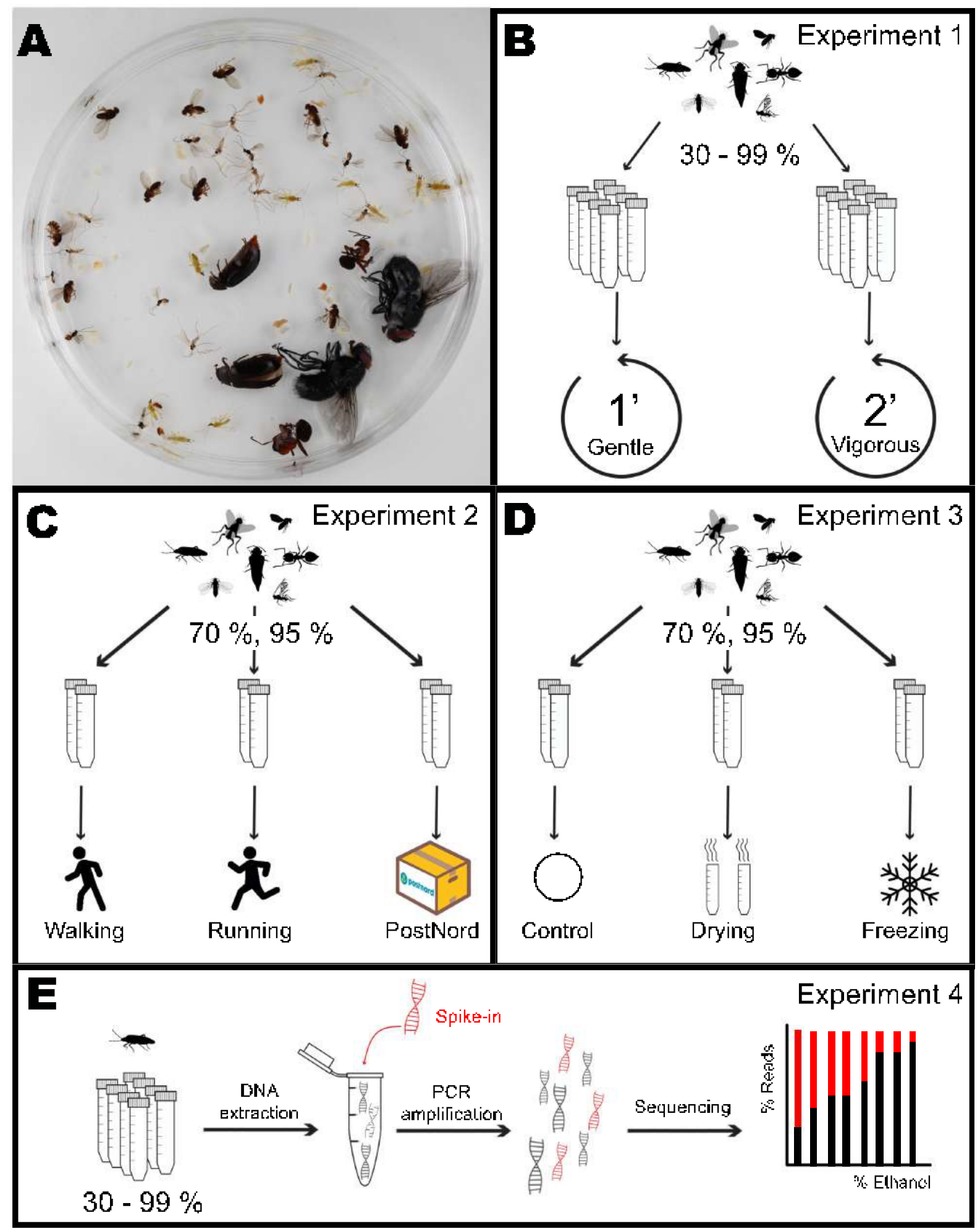

Figure 1. Each mock community sample (A) was assigned to an ethanol concentration and experiment. In Experiment 1 (B), five tubes for each of eight ethanol concentrations (30, 50,70, 80, 90, 95, 97, and 99 \%) were subjected to Gentle or Vigorous shaking. Inn Experiment 2 (C), three tubes for each of two ethanol 
661

662

663

664

665

666

667

668

669

670

671

672

concentrations $(70,95 \%)$ were either carried by a walking or a running experimenter or sent by the Swedish national post service (PostNord) in two different parcels. In Experiment 3 (D), four tubes for each of two ethanol concentrations (70,95\%) were shaken under a Gentle regime after being either dried, frozen or left as control. After each treatment, the number of appendages lost by every individual was scored. In Experiment 4 (E), DNA was extracted from one individual of each species from every tube in treatment Gentle from Experiment 1, mixed with a known quantity of synthetic DNA, PCR amplified and sequenced, and the proportion between insect DNA and synthetic DNA calculated as a measure of DNA degradation.

(1)

(1)

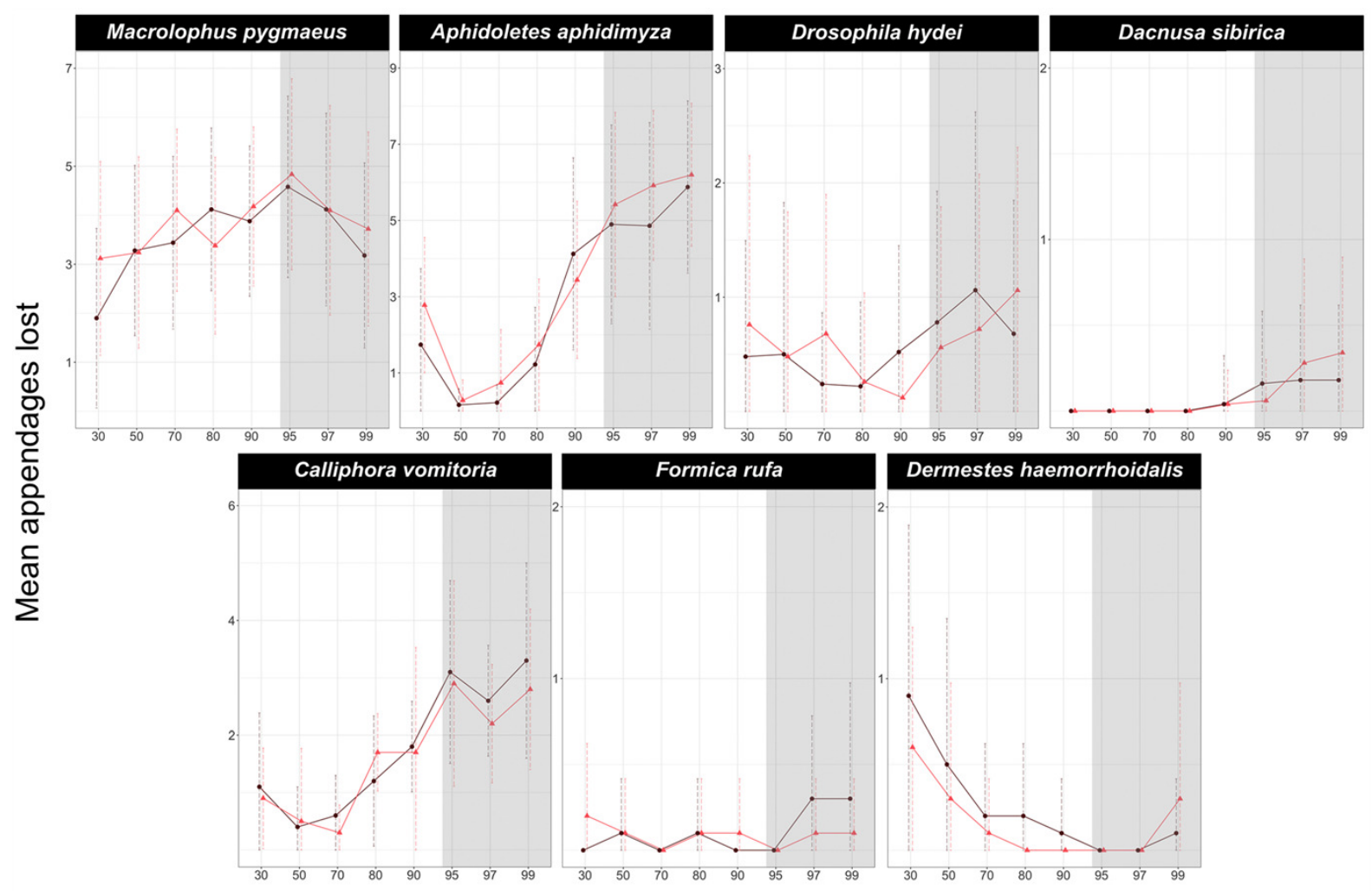

Concentration

673

674 Figure 2 Effect of ethanol concentration on the number of appendages lost by each species. Dark purple

675 circles represent the Gentle shaking regime while bright red triangles represent the Vigorous regime. The 676 shadowed area corresponds to the ethanol concentrations in which DNA is optimally preserved according 677 to literature.

678 
679

680

681

682

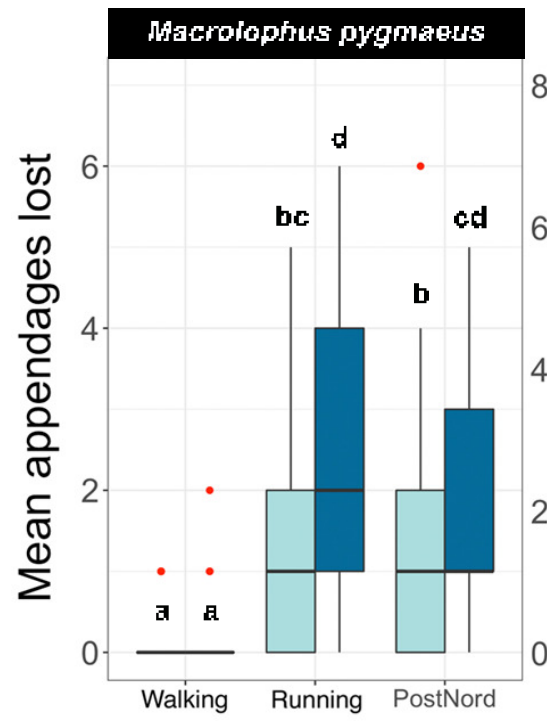

\section{Aphidoletes aphidimyza}

Drosophilla hydei
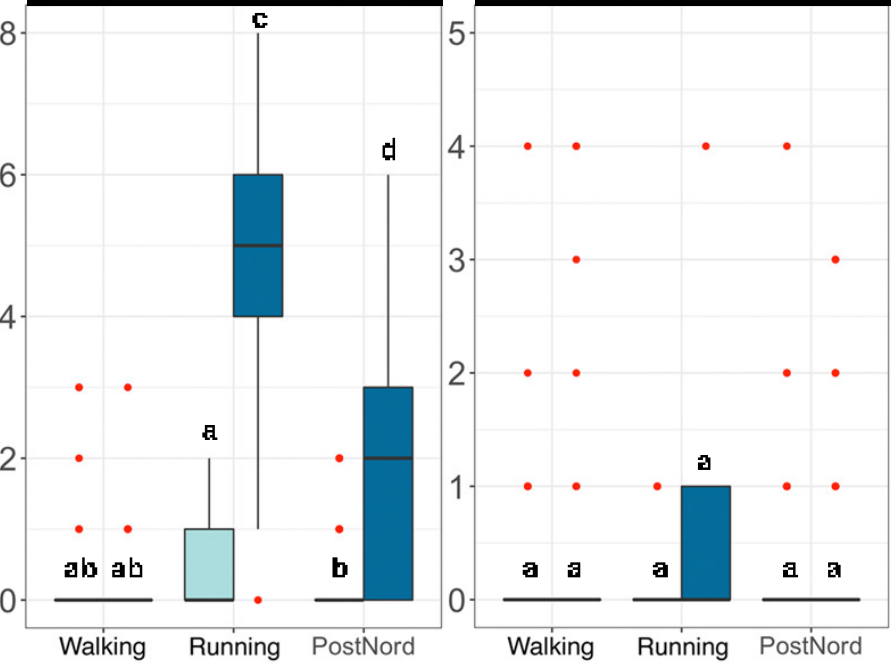

683

Treatment

684 Figure 3 Effect of handling regimes and transport on the number of appendages lost by each species at 685 two different concentrations of ethanol. Light blue boxes represent samples kept in $70 \%$ ethanol and dark 686 blue samples kept in $95 \%$ ethanol. Red dots indicate outliers, and letters indicate groups of the Tukey 687 Test of pairwise comparisons.

688

689

690

691 


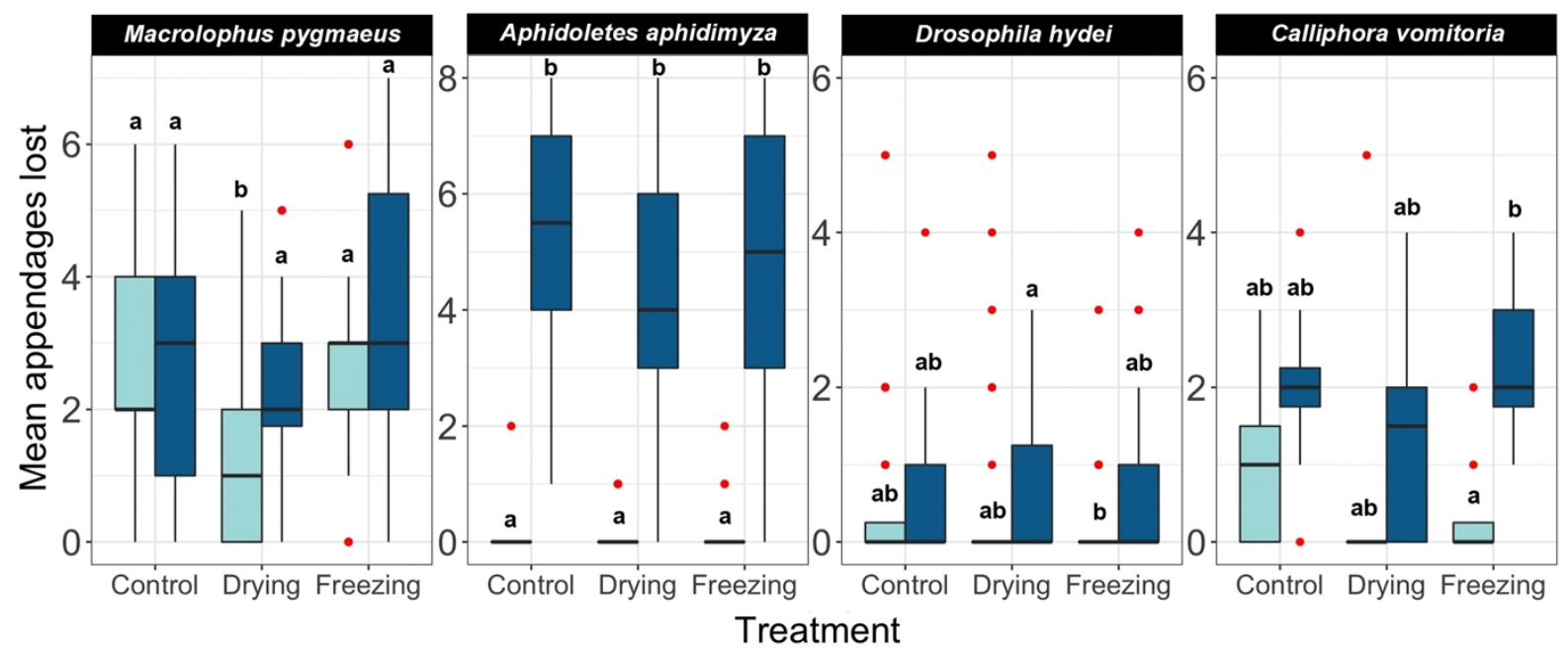

693 Figure 4 Effect of different pre-treatments on the number of appendages lost by each species at two different concentrations of ethanol. All samples were shaken under a Gentle regime. Light blue boxes represent samples kept in $70 \%$ ethanol and dark blue boxes represent samples kept in $95 \%$ ethanol. Red dots indicate outliers, and letters indicate groups of the Tukey Test of pairwise comparisons.

697

698

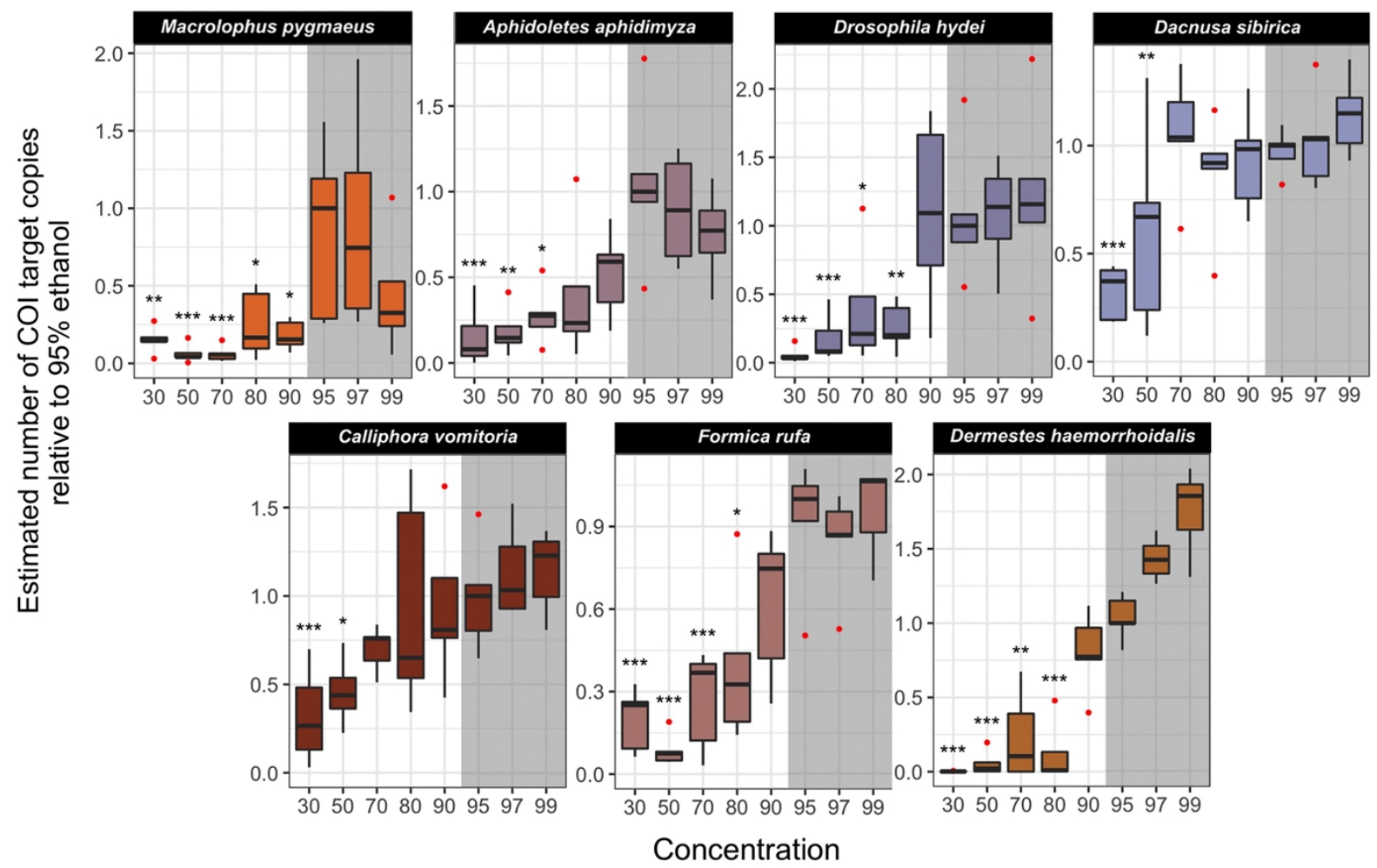

699

700 Figure 5 The estimated number of COI target copies in samples of 7 insect species after one year of storage at room-temperature at eight ethanol concentrations. The numbers were standardized relative to 
$70295 \%$ ethanol concentration median. Barplots were based on 5 or 4 (in four cases) replicates. The 703 concentrations in which the number of COI copies was statistically different from the $95 \%$ ethanol 704 concentration treatment are indicated with asterisks (*** p-value $<0.001, * * \mathrm{p}<0.01, * \mathrm{p}<0.05)$. The 705 shadowed area corresponds to the ethanol concentrations in which DNA is optimally preserved according 706 to literature. 


\section{Table $\mathbf{1}$ (on next page)}

Effect of Concentration, treatment and their interaction in the number of lost appendages for each species in Experiment 1.

F. rufa did not produce enough data points to fit a model. Shown are Type III test of fixed effects if the model described. Asterisks indicate levels of significance: * $p$-value $<0.05$; ** $p$ value $<0.01 ; * * *$ p-value $<0.001$ 
1

\begin{tabular}{|c|c|c|c|}
\hline Effect & $\chi^{2}$ & $\overline{\text { Df }}$ & p-value \\
\hline \multicolumn{4}{|l|}{ Macrolophus pygmaeus } \\
\hline Concentration & 35.0614 & 7 & $0.0001 * * *$ \\
\hline Treatment & 1.7642 & 1 & 0.1841 \\
\hline Concentration:Treatment & 12.4535 & 7 & 0.0866 \\
\hline \multicolumn{4}{|l|}{ Aphidoletes aphidimyza } \\
\hline Concentration & 170.2500 & 7 & $0.0001 * * *$ \\
\hline Treatment & 4.0480 & 1 & $0.0442 *$ \\
\hline Concentration:Treatment & 18.350 & 7 & $0.0104 *$ \\
\hline \multicolumn{4}{|l|}{ Drosophila hydei } \\
\hline Concentration & 10.1877 & 7 & 0.1781 \\
\hline Treatment & 5.3045 & 1 & $0.0212 *$ \\
\hline Concentration:Treatment & 15.7197 & 7 & $0.0278 *$ \\
\hline \multicolumn{4}{|l|}{ Dacnusa sibirica } \\
\hline Concentration & 4.3189 & 7 & 0.7424 \\
\hline Treatment & 0.0000 & 1 & 1.0000 \\
\hline Concentration:Treatment & 3.9310 & 7 & 0.7877 \\
\hline \multicolumn{4}{|l|}{ Calliphora vomitoria } \\
\hline Concentration & 24.5232 & 7 & $0.0009 * * *$ \\
\hline Treatment & 0.6392 & 1 & 0.4239 \\
\hline Concentration:Treatment & 2.3005 & 7 & 0.9413 \\
\hline \multicolumn{4}{|l|}{ Dermestes haemorrhoidalis } \\
\hline Concentration & 11.5213 & 7 & 0.1174 \\
\hline Treatment & 0.3203 & 1 & 0.5714 \\
\hline Concentration:Treatment & 1.6935 & 7 & 0.9748 \\
\hline
\end{tabular}

2 


\section{Table 2 (on next page)}

Effect of Concentration, treatment and their interaction in the number of lost appendages for each species in Experiment 2.

Only M. pygmaeus, A. aphidimyza and D. hydei produced enough data points to fit the model. Shown are Type III test of fixed effects if the model described. Asterisks indicate levels of significance: $* p$-value $<0.05$; $*$ p-value $<0.01$; $* * p$-value $<0.001$. 


\begin{tabular}{lrrr}
\hline Effect & \multicolumn{1}{c}{$\chi^{2}$} & Df & \multicolumn{1}{c}{ p-value } \\
\hline Macrolophus pygmaeus & & & \\
Concentration & 0.0000 & 1 & 1.0000 \\
Treatment & 19.3886 & 2 & $0.0006^{* * *}$ \\
Concentration:Treatment & 0.8273 & 2 & 0.6612 \\
Aphidoletes aphidimyza & & & \\
Concentration & 0.5238 & 1 & 0.4692 \\
Treatment & 11.5596 & 2 & $0.0031^{* *}$ \\
Concentration:Treatment & 21.5104 & 2 & $0.0002 * * *$ \\
Drosophila hydei & & & \\
Concentration & 0.9152 & 1 & 0.3387 \\
Treatment & 3.0386 & 2 & 0.2189 \\
Concentration:Treatment & 1.6271 & 2 & 0.4433 \\
\hline
\end{tabular}

1

2

3

4

5

6

7

8

9

10

11

12

13

14 


\section{Table 3 (on next page)}

Effect of Concentration, treatment and their interaction in the number of lost appendages for each species in Experiment 3.

$D$. haemorrhoidalis, F. rufa and D. sibirica did not produced enough data points to fit the model. Shown are Type III test of fixed effects if the model described. Asterisks indicate levels of significance: $* p$-value $<0.05 ; * *$-value $<0.01 ; * *$-value $<0.001$. 
1

\begin{tabular}{lrrr}
\hline Effect & \multicolumn{1}{c}{$\chi^{2}$} & Df & \multicolumn{1}{c}{ p-value } \\
\hline Macrolophus pygmaeus & & & \\
Concentration & 0.1984 & 1 & 0.6560 \\
Treatment & 21.4882 & 2 & $0.0002 * * *$ \\
Concentration:Treatment & 8.5936 & 2 & $0.0136 *$ \\
Aphidoletes aphidimyza & & & \\
Concentration & 40.8062 & 1 & $0.0001 * * *$ \\
Treatment & 1.1408 & 2 & 0.5653 \\
Concentration:Treatment & 1.5768 & 2 & 0.4546 \\
Drosophila hydei & & & \\
Concentration & 0.6375 & 1 & 0.4246 \\
Treatment & 6.0051 & 2 & $0.0496 *$ \\
Concentration:Treatment & 6.3404 & 2 & $0.0419 *$ \\
Calliphora vomitoria & & & \\
Concentration & 0.1544 & 1 & 0.6943 \\
Treatment & 4.5955 & 2 & 0.1005 \\
Concentration:Treatment & 6.1628 & 2 & $0.0459 *$ \\
\hline
\end{tabular}

2

3

4

5

6

7

8

9

10

11

12

13

14 


\section{Figure 1}

Overview of the experimental design.

Each mock community sample (A) was assigned to an ethanol concentration and experiment. In Experiment 1 (B), five tubes for each of eight ethanol concentrations (30, 50,70, 80, 90, 95, 97, and $99 \%$ ) were subjected to Gentle or Vigorous shaking. Inn Experiment 2 (C), three tubes for each of two ethanol concentrations (70,95\%) were either carried by a walking or a running experimenter or sent by the Swedish national post service (PostNord) in two different parcels. In Experiment 3 (D), four tubes for each of two ethanol concentrations (70, $95 \%$ ) were shaken under a Gentle regime after being either dried, frozen or left as control. After each treatment, the number of appendages lost by every individual was scored. In Experiment 4 (E), DNA was extracted from one individual of each species from every tube in treatment Gentle from Experiment 1, mixed with a known quantity of synthetic DNA, PCR amplified and sequenced, and the proportion between insect DNA and synthetic DNA calculated as a measure of DNA degradation. 


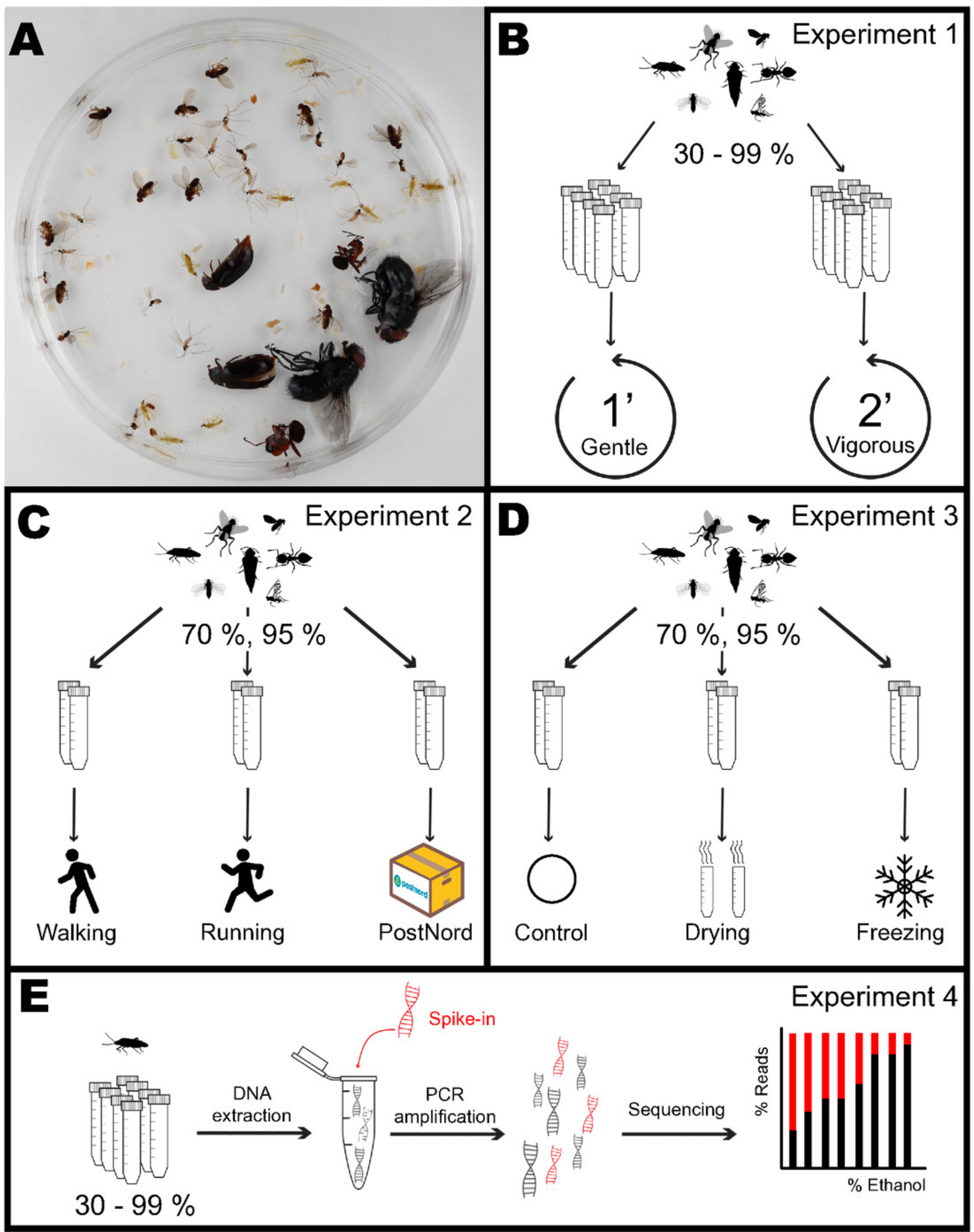


Figure 2

Effect of ethanol concentration on the number of appendages lost by each species.

Dark purple circles represent the Gentle shaking regime while bright red triangles represent the Vigorous regime. The shadowed area corresponds to the ethanol concentrations in which DNA is optimally preserved according to literature.

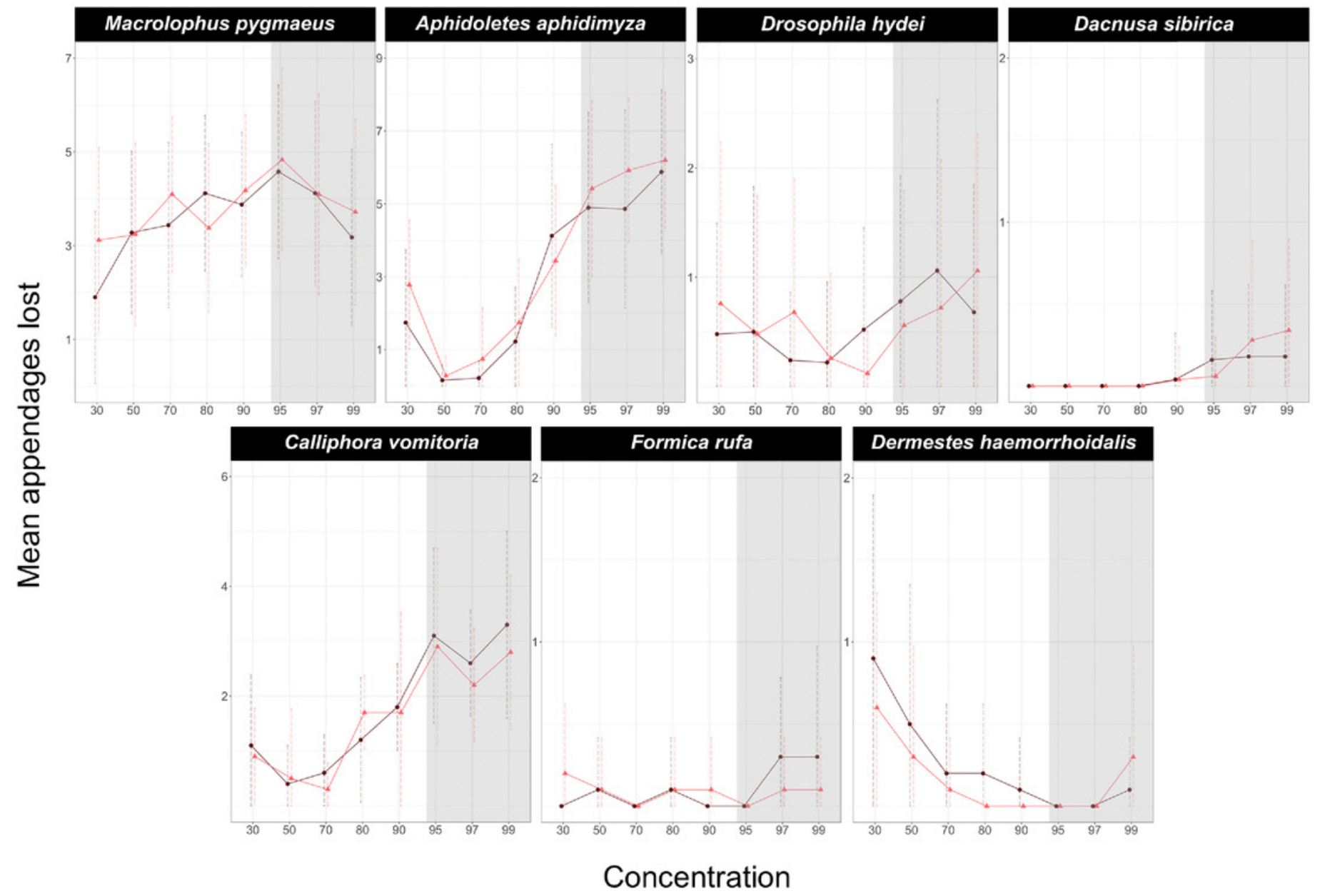


Figure 3

Effect of handling regimes and transport on the number of appendages lost by each species at two different concentrations of ethanol.

Light blue boxes represent samples kept in $70 \%$ ethanol and dark blue samples kept in $95 \%$ ethanol. Red dots indicate outliers, and letters indicate groups of the Tukey Test of pairwise comparisons.

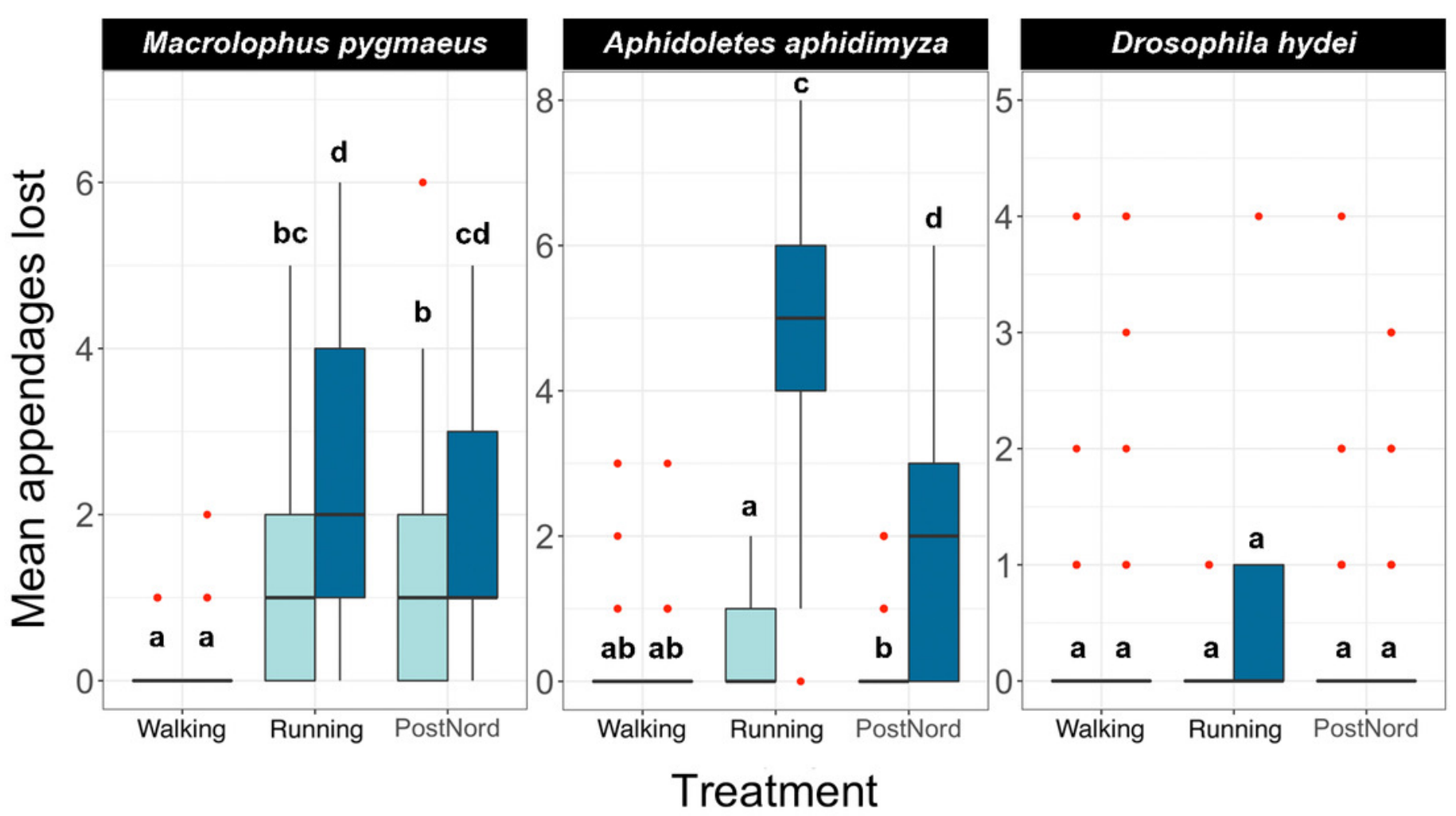




\section{Figure 4}

Effect of different pre-treatments on the number of appendages lost by each species at two different concentrations of ethanol.

All samples were shaken under a Gentle regime. Light blue boxes represent samples kept in $70 \%$ ethanol and dark blue boxes represent samples kept in $95 \%$ ethanol. Red dots indicate outliers, and letters indicate groups of the Tukey Test of pairwise comparisons.

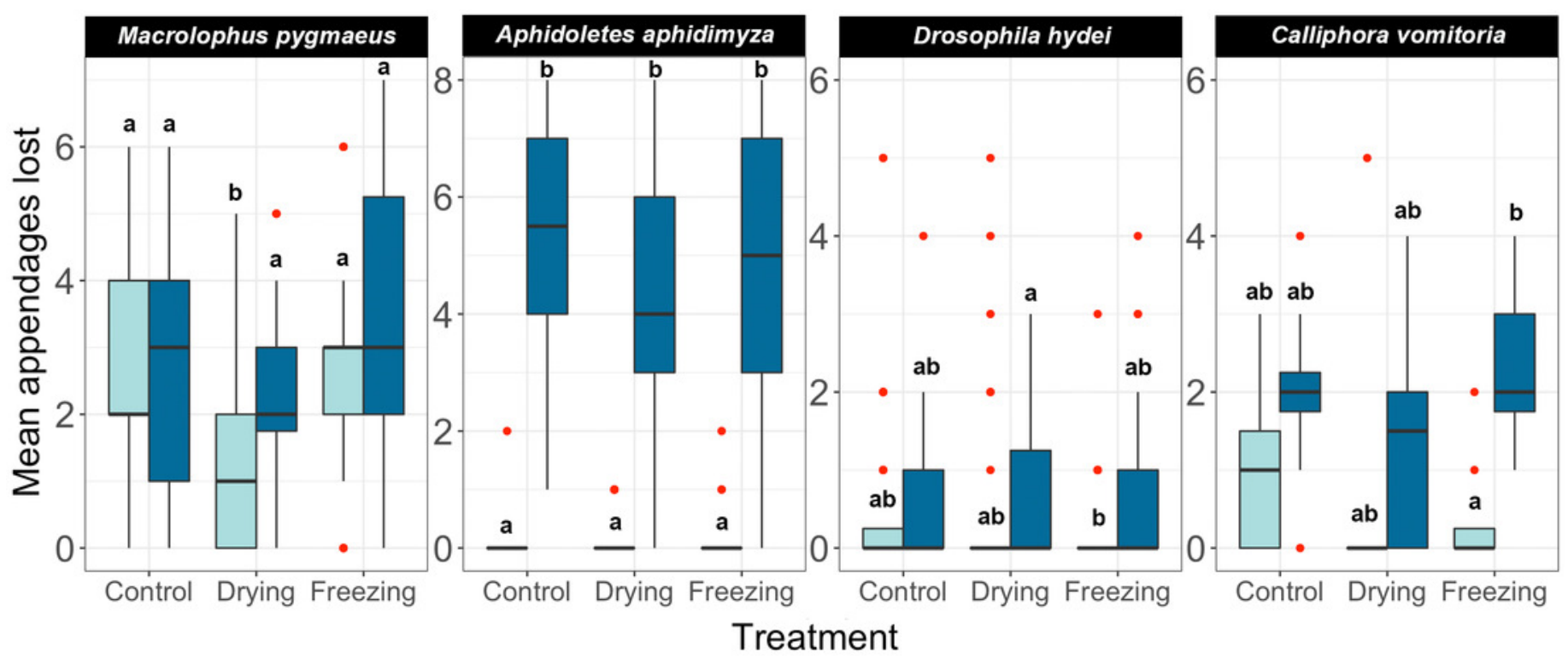




\section{Figure 5}

Estimated number of COI target copies in each species after one year of storage at room-temperature at eight ethanol concentrations.

The numbers were standardized relative to $95 \%$ ethanol concentration median. Barplots were based on 5 or 4 (in four cases) replicates. The concentrations in which the number of COI copies was statistically different from the $95 \%$ ethanol concentration treatment are indicated with asterisks (*** p-value $<0.001, * * p<0.01, * p<0.05$ ). The shadowed area corresponds to the ethanol concentrations in which DNA is optimally preserved according to literature.

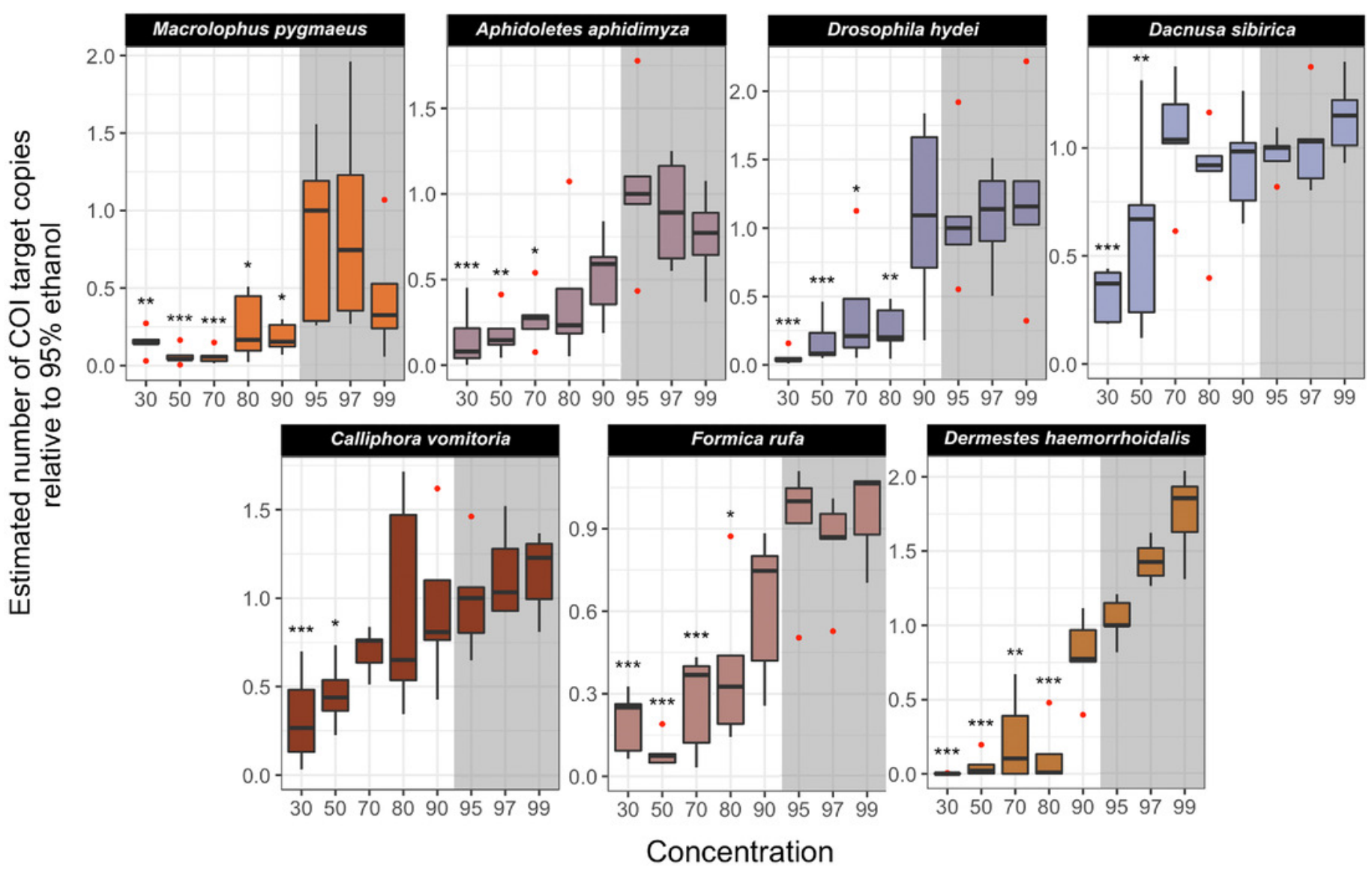

